Faculdade de Ciências Econômicas UFRGS
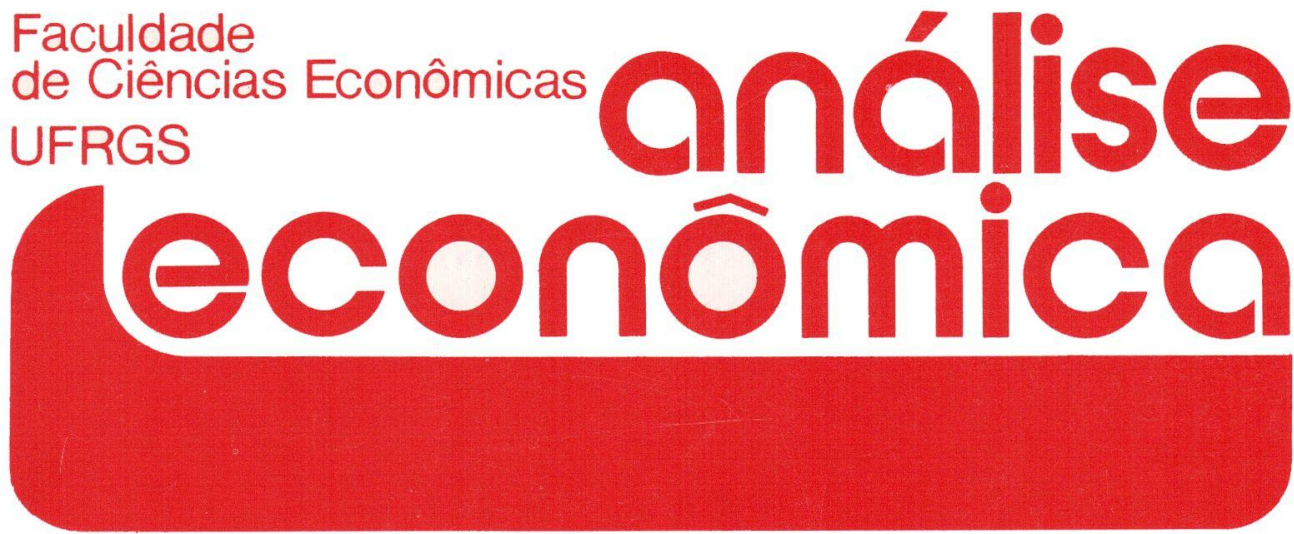

- INDEXAÇĀo SALARIAL: UMA ABORDAGEM MACROECONÓMICA Jo Anna Grav

- AJUSTE NO EMPREGO E PRODUTIVIDADE NA DECADA DE OITENTA

Carlos Antônio Luque José Paulo Zeeteno Chahad

- O CONSUMIDOR KEYNESIANO Marcelo Córtes Neri

- HETEROGENEIDADE DO TRABALHO E TAXA DE LUCRO EM MARX

Francisco Cribari Neto

- ECONOMIAS DE ESCALA: UMA REVISĀO Jesiel de Marco Gomes

- Concentraçấo bancária no BRASIL

Marcelo Resende

- NOYOS RUMOS PARA O SETOR ELETRICO NO BRASIL

Adriano Pires Rodrigues

Eduardo da Cunha Vianna

- OFERTA E DEMANDA DE FRANGO DE CORTE NO BRASIL

Narciso Gonçalves de Castro et alii

- ANÁLISE ECONÔMICA DA IRRIGAÇÃo DO MILHO

Lúcia M Schirmer

Juvir Luiz Mattuella

- REFLORESTAMENTO NO BRASIL. Carlos José Caetano Bacha

- ESCOLHA DE TECNOLOGIA EM ESTRUTURA DE PRINCIPAL AGENTE Kyle D. Kauffma:

- A QUESTÁo DEMOGRÁfica e A PRAXEOLOGIA

Anton Karl Biedermann et alii

UNIVERSIDADE FEDERAL DO RIO GRANDE DO SUL

Reitor: Prof. Tuiskon Dick

FACULDADE DE CIÉNCIAS ECONÔMICAS

Diretora: Prof ${ }^{\text {a }}$ Yeda Rorato Crusius.

CENTRO DE ESTUDOS E PESQUISAS ECONÔMICAS

Diretor: Reinaldo Ignacio Adams

DEPARTAMENTO DE CIÉNCIAS ECONÔMICAS

Chefe: Prof. Fernando Ferrari Filho

CURSO DE POS-GRADUAÇÄO EM ECONOMIA

Coordenador: Prof. Nali de Jesus de Souza

CURSO DE PÓS-GRADUAÇĀO EM ECONOMIA RURAL

Coordenador: Prof. Atos Freitas Grawunder

CONSELHO EDITORIAL: Achyles Barcelos da Costa, Aray Miguel Feldens, Atos Freitas Grawunder, Carlos Augusto Crusius, Ernani Hickmann, João Rogério Sanson, Juvir Luiz Mattuella, Maria Imilda da Costa e Silva, Nali de Jesus de Souza, Nuno Renan Lopes de Figueiredo Pinto, Otilia Beatriz Kroeff Carrion, Otto Guilherme Konzen, Paulo Alexandre Spohr, Pedro Cezar Dutra Fonseca, Reinaldo Ignacio Adams, Roberto Camps Moraes, Valter José Stülp, Yeda Rorato Crusius, David Garlow (Wharton Econometrics Forecasts Association, E.U.A.), Edgar Augusto Lanzer (UFSC), Eleutério F.S. Prado (USF), Fernando Holanda Barbosa (FGV/RJ), Gustavo Franco (PUC/RJ), Joaquim Pinto de Andrade (UnB), Juan H. Moldau (USP), Werner Baer (Univ. de Illinois, E.U.A.).

COMISSĀO EDITORIAL: Atos Freitas Grawunder, Pedro Cezar Dutra Fonseca, Reinaldo Ignacio Adams e Roberto Camps Moraes.

EDITOR: Nali de Jesus de Souza

SECRETARIA: Maria Ivone de Mello (normalização), Vanete Ricacheski (revisão de textos).

FUNDADOR: Prof. Antônio Carlos Santos Rosa

Os materiais publicados na revista Análise Econômica são de exclusiva responsabilidade dos autores. É permitida a reprodução total ou parcial dos trabalhos, desde que seja citada a fonte.

Aceita-se permuta com revistas congêneres. Aceitam-se, também, livros para divulgação, elaboração de resenhas ou recenșões.

Toda correspondência, material para publicação, assinaturas e - permutas devem ser dirigidos ao seguinte destinatário:

\section{PROF. NALI DE JESUS DE SOUZA}

Revista Análise Econômica

Av. João Pessoa, 52

CEP 90040-000 - PORTO ALEGRE (RS), BRASIL

Telefones: (051) 228-1633 - 224-6024 ramais 3440 e 3348

Fax: (051) 225-1067 


\title{
HETEROGENEIDADE DO TRABALHO E TAXA DE LUCRO EM MARX*
}

\author{
Francisco Cribari Neto**
}

\section{SINOPSE}

O objetivo do presente artigo 6 o de analisar o comportamento uinfmico da taxa de lucro vem uma estrutura marxista uma vez que o capital humano $\varepsilon$ incorporado como fator de produçlo. Neste novo contexto, deriva-se um conjunto de condiḉes suficientes para um comportamento crescente náo só da taxa de lucro, mas também da taxa salarial ao longo do processo de acumulaçăo.

\section{INTRODUÇĀO}

Em sua análise, Marx chegou a prever um aprofundamento dos conflitos de classe determinado pela ação dos capitalistas em busca de ùma elevação da exploração como medida compensatória - ou parcialmente compensatória - à tendência declinante da taxa de lucro. Tal declínio seria causado pelas elevaçōes na composição orgânica do capital, caracterizadoras do processo de acumulação.

Esta conclusão, contudo, tem sofrido intensos quesiionarisentos. Em um célebre artigo, Okishio (1961) utiliza um modelo n-setorial de indústrias de produto único, sem capital fixo e com o trabalho como o único insumo não produzido para mostrar que existe uma tendência oposta no comportamento dinâmico da taxa de juros, i.e., existe uma tendência crescente desta taxa ao longo do processo de acumulaçãu. Tal

* Agradeço os comentários e sugestőes de Kyle Dean Kauffman, Marai da Conceiçăo Sampaio de Sousa e, em especial, Brian David Ballard. Os ertos e omissues remanescentes 8 tio (infelizmente) meus.

** Doutorando em Economia da Universidade de Illinois, EUA.

\begin{tabular}{|l|l|l|l|l|}
\hline ANÅLISE ECONÔMICA & ANO 10 & NN 17 & MARÇO/92 & P.49-57 \\
\hline
\end{tabular}


resultado é conhecido na literatura por Teorema de Okishio.1 Woods (1985) relaxa a hipótese de ausência de capital fixo e chega às mesmas conclusōes. Roemer (1981) detalha a análise, mostrando os casos onde a taxa de lucro e crescente e apresentando uma abordagem mais geral para a existência de capital fixo em um modelo de Von Neumann.

Entretanto, todas estas análises seguem o figurino marxista de suposição de homogeneidade do fator trabalho. Assim sendo, são limitadas pelo curso recente do desenvolvimento capitalista, onde os investimentos em capital humano tem-se tornado cada vez mais intensos e significativos. A proporção do trabalho especializado no trabalho total tem crescido acentuadamente em perfodos mais recentes, principalmente naqueles parses que atravessam processos mais intensos de crescimento e desenvolvimento. 2

Neste sentido, Mankiw, Romer e Weil (1990) salientam:

Economists have long stressed the importance of human capital to the process of growth... Including human capital can potentially alter either the theorical modelling or the empirical analysis of economic growth. $\checkmark$

Uma outra limitação do sistema marxista ê a falta de referência à geração de conhecimenius. De acordo com Chakravarty (1987):

[G]iven the contemporary trends in technology, it is important to allow for a theory of 'knowledge' production and dissemination, an area where interesting work is being done by contemporary economists working outside the Marxist framework.

Quais as implicações sobre a tendência decrescente da taxa de lucro marxista uma vez que estas limitaçōes são superadas? O propósito do present $\epsilon$ artigo $\in$ o de fornecer uma resposta a tal pergunta.

A próxima seção expẽe sumariamente o entendimento marxista de que há um declf́nio gradual da taxa de lucro 30 decorrer do processo de acumulação. A seçaio três apresenta uma discussão accrca da compatibilidade do trabalho qualificado com a Teoria do Valor. À seção quatro cabe a análise do comportamento da taxa de um lucro em um contexto de heterogeneidade do trabalho. Por fim, a última seção sumariza as conclusōes do presente trabalho.

\section{A TAXA DECRESCENTE DE LUCRO}

Uma das conseqüências mais importantes do processo de acumula-

1 Para uma prova simplificada, ver Bidard (1988).

2 A desconsideraçăo do capital humano como fator de produçăo já causou embaraços teóricos significativos, como, e.g., o paradoxo de Leontief no campo da Economia.Internacional. 
ção para Marx era uma tendência à elevação na composição orgânica do capital. Como apenas o trabalho gera valor - de acordo com a Teoria do Valor-Trabalho, por ele encampada -, quando uma dada quantidade deste fator se combina a uma quantidade cada vez maior de capital constante, dada uma taxa de exploração, cria-se uma tendência a um declínio gradual da taxa de lucro. Isto pode ser visto facilmente quando se nota que:

$$
I I=\frac{S}{C+V}=\frac{S}{V}\left(l-\frac{C}{C+V}\right)=e(I-k)
$$

onde II representa a taxa de lucro, $\mathrm{S}$ a mais-valia, $\mathrm{C}$ o capital constante, $\mathrm{V}$ o capital variável e a taxa de exploração e $\mathrm{k}$ a composição orgânica do capital.

Os capitalistas poderiam lançar mão de mecanismos compensatórios, tal como a exploração mais intensa do comércio externo, a fim de tentar impedir o declínio do lucro total. Todavia, a aludida tendência não estaria revertida. A sua realização seria apenas uma questão de tempo.

Dentre várias, três críticas podem ser desde logo enumeradas. A primeira refere-se ao fato de que Marx ignora uma tendência ao barateamento do capital constante ao longo do processo de acumuiação, o que constitui um fator de elevação da lucratividade. $3 \mathrm{Em}$ segundo lugar, Marx toma as variações ná composição orgânica do capital como sendo independentes das variações na taxa de exploração. Caso um aumento daquela implique um aumento desta, não se pode precisar o efeito líquido A rationale desta interdependência encontra-se na tendência à elevação da produtividade do trabalho no curso do processo de acumulação. Este efeito torna-se mais visfvel quando se separa a mão-de-obra em qualificada e não-qualificada, assumindo-se que a primeira seja uma fonte de desenvolvimento tecnológico e, assim, responsável por consideráveis melhorias de eficiência. 4 Por fim, Marx ignora o fato de que o aparecimento de uma nova técnica não obriga a sua adoção, que somente deverá ocorrer frente à perspectiva de elevação na lucratividade. A este respeito, aduz Simonsen (1984):

Por que os capitalistas resolvem encampar essas novas tecnicas que só servem para baixar a taxa de lucro da economia, eis um enigma a decifrar. Afinal, a descoberta de um metodo de produçăo năo obriga nenhuma empresa a adotá-lo.

Uma possível alegação seria a de que a motivação para a adoção de

3 Um parecerista chamou a atençăo para o fato de que năo é consensualmente aceito que Marx ignore tal tendencia.

4 Ver seçá quatro do presente trabalho. 
uma nova técnica decorreria de uma busca de redução de custos. Contudo, neste Caso Roemer (1981) salienta:

If a technical change is introduced by capitalists only when it is cost reducing at current prices, then the equilibrium rate of profit will rise.

\section{O TRABALHO QUALIFICADO E A TEORIA DO VALOR}

Desde a publicação de $O$ Capital muitos autores têm questionado se a existência de trabalho qualificado e compatf́vel com a Teoria do Valor marxista. Para que esta compatibilidade exista é necessário, em primeiro lugar, que o trabalho qualificado possa ser reduzido a trabalho nāo-qualificado, simples. Em segundo lugar, é necessário que os valores resultantes de tal transformação independam das taxas salariais pagas. Tal polêmica se estabeleceu na literatura com a designação de o problema da redutibilidade.

Muito embora não haja uma posição estabelecida,5 Rowthorn (1982), retomando Hilferding, mostrou que é possível estabelecer uma forma de redução que atenda ao requisito de independência entre valores e taxas saláriais. Para tanto, tratou o setor educacional como um setor produtivo, com as características de qualquer outro, e assumiu que o trabalho não-qualificado adquire qualificação através deste setor. Desta forma, o trabalho qualificado pode ser decomposto em uma parcela nãoqualificada e em uma parcela de qualificação adquirida. Esta última pode ser decomposta em trabalho nāo-qualificado e em trabalho qualificado, presentes no processo "produtor" de educação. Esta parcela de trabalho qualificado, por sua vez, pode igualmente ser decomposta em trabalhos de ambos os tipos de um momento imediatamente anterior Retrocedendo-se, assim, indefinidamente no tempo tem-se uma redutibilidade perfeita do trabalho qualificado a trabalho simples; sem qualificação. $O$ que e fundamental neste processo redutor de Rowthorn é que ele independe das taxas salariais pagas a ambas as categorias.

O restante do artigo assume a validade da solução acima apresentada.

\section{O TRABALHO QUALIFICADO COMO CATEGORIA FATORIAL E A TENDÊNCIA DECLINANTE DA TAXA DE LUCRO}

Considere-se uma estrutura departamental marxista, onde o primeiro departamento produz bens de capital, o segundo produz bens-salário e

5 Ver Elster (1984). 
o terceiro se ocupa da produção de bens para o consumo dos capitalistas. A notação utilizada é a seguinte: $C$ representa o capital constante, $S$ a mais valia, $Y$ o produto total, Vn o trabalho não-qualificado e Vq o trabalho qualificado. Assim, o produto de cada setor pode ser escrito como:

$$
Y_{i}=C_{i}+V n_{i}+V q_{i}+S_{i}
$$

onde $i=1,2,3$ denota o respectivo departamento. Normalizando-se a equação acima em termos de trabalho não-qualificado, tem-se:

$$
Y_{i}^{*}=1+C_{i}^{*}+V q_{i}^{*}+S_{i}^{*}
$$

onde o asterisco $\left(^{*}\right)$ indica que todas as variáveis foram divididas por $V n_{i}$.

As variáveis $S^{*}$ e $C^{*}$ são aproximações aos conceitos marxistas de taxa de exploração e de composição orgânica do capital, respectivamente. A variável $V q^{*}$, por sua vez, representa a razão dos trabalhos qualificado e não-qualificado. Tal variável pode ser chamada, a título de analogia, composição do trabalho.

O próximo passo é definir a taxa de lucro neste novo contexto:6

$$
I I=\frac{S^{*}}{1+C^{*}+V q^{*}}
$$

onde $S^{*}=S\left(V q^{*}\right)$ e $\varepsilon$ assumido que $s^{*}>0$, ou seja, que elevações na composição orgânica do trabalho conduzem a elevaçôes na taxa de exploração. Uma possível justificação para que se assuma tal comportamento reside no fato de que o trabalho qualificado es uma fonte geradora de progresso tecnologico, o que conduz a elevaçōes na produtividade do trabalho simples. De acordo com Schultz (1990):

In the modernizing economy, most of the increases in income originate out the proliferation of human capital... People at each skill level are more productive in high than low human capital environments. Human capital enhances the productivity of both labor and phisical capital.

De (4) obtém-se:

$$
\begin{aligned}
& \frac{\partial I I}{\partial C^{*}}=-\frac{S^{*}}{\left(Y^{*}-S^{*}\right)^{2}} \\
& \frac{\partial I I}{\partial V q^{*}}=\frac{S^{\prime}}{Y^{*}-S^{*}}-\frac{S^{*}}{\left(Y^{*}-S^{*}\right)^{2}}
\end{aligned}
$$

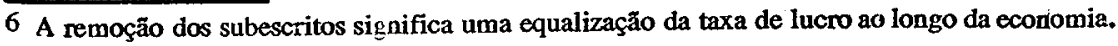


De (5) nota-se que $\partial I I / \partial C^{\circ}<O$, o que está em compatibilidade com a análise marxista tradicional. Contudo, o efeito de variações na composição orgânica do trabalho sobre a taxa de lucro não é tão claro. Nota-se, entretanto, que ambas as variáveis variarão no mesmo sentido caso:

$$
S^{\prime}>\frac{S^{*}}{Y^{*}-S^{*}}
$$

ou, equivalentemente, se:

$$
S^{\prime}>\frac{S}{Y-S}
$$

Deste modo, nota-se que elevações na composição orgânica do capital conduzem a reduções na taxa de lucro, enquanto o efeito de elevações na composição orgânica do trabalho sobre esta é ambrguo, dependendo de seu impacto sobre a taxa de exploração.

Deslocando a análise para um contexto dinâmico, a taxa de lucro pode ser escrita como:

$$
I I(t)=f\left(C^{*}(t), V q^{*}(t), S^{*}\left(V q^{*}(t)\right)\right)=h\left(C^{*}(t), V q^{*}(t)\right)
$$

onde a hipótese clássica de que $h_{l} \equiv \partial \Pi / \partial C^{*}<O$ e assumida, ou seja, assume-se que elevações na composição orgânica do capital conduzem a um declínio na lucratividade. Uma segunda hipótese é que $h_{2} \equiv \partial \Pi$ $/ \partial \mathrm{Vq}^{*}>0$, isto $\mathcal{E}$, aumentos do trabalho qualificado em relação ao trabalho não-qualificado tendem a elevar a lucratividade. Note-se que, aqui, está-se assumindo que $s^{\prime}>S /\left(Y-S+S^{*} /\left(Y^{*}-S^{*}\right)\right.$. Esta ultima hipótese pode ser justificada à luz da estreia relação existente entre capital humano e eficiência microeconômica. ${ }^{7}$

Pode-se ainda assumir que ambas as composições orgânicas aumentem ao longo do processo de acumulação, ou seja, $d C * / d t>0$ e $d V q^{*} / d t>0$.

Sendo $w$ a taxa salarial, pode-se igualmente escrever. ${ }^{8}$

$$
w(t)=g\left(C^{*}(t), V q^{*}(t), S^{*}\left(V q^{*}(t)\right)\right)=l\left(C^{*}(t), V q^{*}(t)\right)
$$

assumindo que $l_{1} \equiv \partial w / \partial C^{*}<O$ e $I_{2} \equiv \partial w / \partial V q^{*}>0$. Ou seja, assume-se que elevaçốes na composição orgânica do capital possuem um

7 Esta argumentaçăo está desenvolvida em Fonseca (1989).

8 Aqui $w$ representa a taxa salarial do trabalho simples, uma vez que, assumida a redutibilidade de Rowthorn, trabalho qualificado pode ser reduzido a trabalho simples independentemente das taxas salariais. 
efeito depressivo sobre a taxa salarial, ao passo que elevações na composição orgânica do trabalho possuem um efeito expansivo sobre esta taxa. Esta última hipótese merece alguma justificação. $O$ trabalho qualificado constitui, como ressaltado anteriorm:nte, uma fönte de contínuo desenvolvimento tecnologico, o que eleva a produtividade do trabalho nâo-qualificado. Este aumento de produtividade é dividido entre capitalistas e trabalhadores na forma de aumentos nas taxas de lucro e de salario. A fatia de cada classe nesta divisão depende de seu poder de organização e negociação, variando, assim, em cada paŕs. zem:

Sobre esta segunda hipótese, Jorgenson e Fraumeni (1991) adu-

[ I Investments in human beings, like investments in tangible forms of capital such as buildings and industrial equipment, generates a stream of future benefits. Educations is regarded as an investiment in human capital, since benefits accrue to an educated individual over a lifetime of activities.

Tem-se portanto, de (9) e (10):

$$
\begin{aligned}
& \frac{d \Pi}{d t}=h_{1} \frac{d C^{*}}{d t}+h_{2} \frac{d V q^{*}}{d t} \\
& \frac{d w}{d t}=l_{1} \frac{d C^{*}}{d t}+l_{2} \frac{d V q^{*}}{d t}
\end{aligned}
$$

Considere-se primeiramente a equação (11). Nota-se que a taxa de lucro crescerá ao longo do tempo, i.e., $\partial \Pi / \partial t>0$, se e somente se:

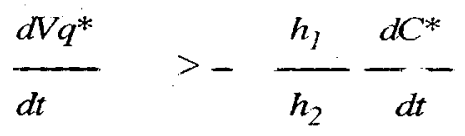

ou seja, caso a taxa de crescimento da composição orgânica do trabalho seja maior que o produto da taxa de crescimento da composição orgânica do capital e đo valor absoluto da razão de efeitos contracionistas e expansionistas do processo de acumulação sobre a taxa de lucro.

No que tange à taxa salarial, a equação (12) revela que esta apresentará uma tendência crescente se e somente se:

$$
\frac{d V q^{*}}{d t}>-\frac{l_{l}}{l_{2}} \frac{d C^{*}}{d t}
$$

ou seja, caso a taxa de crescimento da composição orgânica do ca- 
pital seja superior ao produto da taxa de crescimento da composição orgânica do capital e do valor absoluto da razão de efeitos contracionistas e expansionistas do processo de acumulação sobre a taxa salarial.

As equaçōes (13) e (14) possibilitam duas importantes conclusões. A primeirc é que a taxa de lucro deixa de ter uma tendência necessariamente decrescentc quando se introduz o capital humano como uma categoria fatorial em um sistema marxista básico. Neste contexto, os aumentos de produtividade oriundos do progresso ténico - cuja fonte $\varepsilon$ o trabalho especializado - podem compensar o efeito depressivo sobre a taxa de lucro das elevaçōes na composição orgânica do capital. Mais ainda: é possivel que ambas as taxas salarial e de lucro apresentem um comportamento ascendente ao longo do tempo. Para tanto é necessário que:

$$
\frac{d V^{*} q}{\mathrm{dt}}>m \frac{d C^{*}}{\mathrm{dt}}
$$

Onde $m=\max \left[-h_{1} / h_{2}, l_{1} / 1_{2}\right]>0$. Neste caso, o processo de crescimento e acumulação não se daria em bases conflituais, como queria Marx.

A segunda conclusão das equações acima é que, neste novo contexto, polf́ticas econômicas podem desempenhar um importante papel na preservação do sistema capitalista. Polf́ticas que incentivem a formação de novo capital humano, elevando a taxa de crescimento da composição orgânica do trabalho, elevarão a lucratividade e a taxa salarial da economsia.

\section{CONCLUSÃO}

Uma das peças centrais da análise marxista $\varepsilon$ a tendência declinante da taxa de lucro devido às suias implicaçōes para a superação do modo capitalista de produção. Contudo, o sistema original através do qual esta tendência foi derivada tem-se mostrado por demais simplista, Jadas as características das sociedades capitalistas contemporâneas. Assim, o objetivo do presente trabalho foi o de revisitar esta conclusão marxista procurando superar duas limitaçōes: as ausências de uma teoria da produção de conhecimentos e de capital humano.

Neste sentido, introduz-se heterogeneidade do fator trabalho, e assume-se que o trabalho qualificado seja uma fonte cont́nua de produção 
de conhecimentos e melhorias tecnológicas. $\mathrm{O}$ impacto de tais melhorias sobre a economia se dá via elevação da produtividade da força de trabalho não-qualificada, elevando assim a taxa de exploração.

Neste novo cenário, encontra-se um conjunto de condiçōes suficientes para um comportamento crescente tanto da taxa de lucro quanto da taxa salarial ao longo do tempo. Assim, o processo de acumulação e crescimento não necessariamente precisa dar-se em bases conflituais.

\section{BIBL.IOGRAFIA}

BIDARD, C. The Falling Rate of Profit and Joint Production. Cambridge Journal of Economics, v.12, n.3, p.355-60, 1988.

CHAKRAVARTY, S. Marxist Economics and Contemporary Developing Economies. Cambridge Journal of Economics, v.12, n.1, p.3-22, 1987.

ELSTER, J. Making Sense of Marx. Cambridge: Cambridge University Press, 1984.

FUNSECA, E.G. Liberalismo e Reforma Social: o Legado Utilitarista. São Paulo: IPE/USP, 1989. (Texto para Discussão, n.2).

JORGENSON, D.W. \& FRAUMENI, B.M. The Ontpat of the Education Sector. Cambridge, Mass.: Harvard University, Department of Economics, 1991. (Discussion Paper, n.1543).

MANKIW, W.G.; ROMER, D. \& WEIL, D.N. A Contribution to the Empirics of Economic Growth. Cambridge, Mass.: Harvard University, Department of Economics, 1990 (mimeo).

ROEMER, J.E. Analytical Foundations of Marxian Economic Theory. Cambridge: Cambridge University Press, 1981 .

ROWTHORN, B. Capitalismo, Conflito e Inflaç̄ón. Rìo de Janeiro: Zahar, 1982. Caṕtulo 8, Trabalho Qualificado no Sistema Marxista, p. 211-27.

SCHULTZ, T.W. Restoring Economic Equilibriam. Brasil Blackwell, 1990.

SIMONSEN, M.H. Número Especial Sobre Marx e a Revolução de Von Neumann. Revista Brasileira de Economia, v.38, n.2, abr./jun., 1984.

WOODS, J.E. Okishio's Theorem and Fixde Capital. Metroconomica, v.37, p. 187-97, 1985.

\section{ADSTRACT}

The purpose of this paper is to analyze the dynamic behavior of the rate of profit in a marxian framework, once human capital is incorporated as a production factor. In this context, a set of sufficient conditions for an increasing behavior of the rate of profit and the wage rate along the accumulation process is derived. 